Aus der Wiener Universitätsklinik für Dermatol. u. Syphilidologie .

(Prof. Dr. Gustav Ri ehl.)

\title{
Zur Kenntnis der Leukonychie.
}

\author{
Von
}

\author{
Dr. Theodor Brauns, \\ Spezialarzt in Dessau (Anhalt).
}

(Hiezu Taf. IV.)

So häufig man die Leukopathia unguium in Form der bekannten weißen Fleckchen innerhalb sonst gesunder Nagelplatten findet, die als "Mendacia“, "Flores unguium" etc. bezeichnet werden, so wenig zahlreich sind die Fälle, in denen ausgedehntere oder totale Weißfärbung des sichtbaren Nagels, die sogenannte Leukonychie, beobachtet worden ist.

Demgemäß ist unsere Kenntnis rom Wesen dieser Erkrankung auch noch keine abgeschlossene. Es handelt sich dabei, wie Morison, Unna, Giovannini, Joseph übereinstimmend angeben, um größere Mengen von Luftbläschen in der Nagelsubstanz, für deren Anwesenheit Morison keine Erklärung gibt, Giovannini "abnorme Keratinisation" verantwortlich macht, Unna eine "Strukturverschiedenheit" der Zellen und "stärkere Schrumpfung durch Wasserverlust" annimmt. Joseph sah in den Zellen Keratohyalinlager, und Heidingsfeld behauptet nur Parakeratose ohne Luftinfiltration sei die Ursache dieser Erscheinung.

Betreffs der Ätiologie der Affektion nimmt man Ernährungsstörungen in der Nagelmatrix an (Unna, Joseph, Bielschowsky), doch können die Flores unguium auch durch leichtes Trauma des Nagelfalzes entstehen, wie Heller an sich selbst erlebte. Er glaubt eine besondere Disposition der Nägel in solchen Fällen annehmen zu sollen, allerdings ohne sich des Näheren darüber auszulassen. 
Woher kommt aber die Ernährungsstörung? Giovannini beschuldigt ein typhöses Fieber, Bielschowsky eine multiple Neuritis, Joseph meint, vielleicht trage in seinem ersten Falle ein entzündlicher Prozeß der Matrix die Schuld, im zweiten, später von Forchbeimer bearbeiteten Falle aber sei möglicherweise Rhachitis verantwortlich zu machen. Unna, Lawrence, Bergmann sind der Meinung, daß die Affektion in ihren Fällen angeboren sein dürfte.

In dieser Richtung erscheint der folgende, in der Ambulanz der Wiener dermatologischen Klinik beobachtete Fall ${ }^{1}$ ) von Interosse.

Es handelte sich um ein junges Mädchen, das angeblich nie ernstlich krank gewesen. Vor einigen Jahren hatte sie am rechten Daumen ein Panaritium des Nagelfalzes, infolgedessen der Nagel sich löste und der neu nachgewachsene dauernd deformiert blieb. In Winter 1900/1 erfror sie sich die Hände und es entstanden Beulen, die damals einer Salbenbehandlung allmählich wichen; im folgenden Winter wiederholte sich die Erfrierung, ebenfalls unter Pernionenbildung; im letzten Winter aber trat eine allgemeine diffuse Blaurotfärbung der Hände auf. Nach Abheilung der erstmaligen Erfrierung soll die jetzt bestehende Verdickung der Oberhaut in beiden Handflächen begonnen haben. Die Haut sei allmählich derber und fester geworden und hindere sie jetzt daran, die Finger ganz auszustrecken. Seit ca. 2 Monaten etwa fiel ihr nun eine weißliche Verfärbung der Nägel auf, die vom Grunde nach der Fingerkuppe zu langsam anwuchs. Auf einige weiße Punkte und Streifen in den vorderen, zum Teil noch normalen Nägelanteilen aufmerksam gemacht, erklärte sie dieselben nie beachtet zu haben. Irgend welche abnormen Empfindungen in den Fingern hat sie nicht beobachtet. Die Füße sind niemals erfroren gewesen. In der Familie soll der Vater ebenfalls stärker verhornte Oberhaut in den Flachhänden haben, doch behauptet Patientin, daß diese bei ibm sicherlich nur infolge der Arbeit gekommen sei, während sie selbst negiert, je schwere Arbeit getan zu haben. Die anderen Familienmitglieder sind gesund und haben weder eine gleiche Erkrankung der Flachhände, noch der Nägel. Angaben über die Großeltern sind nicht zu erbalten.

Die Patientin ist von untersetzter Statur, kräftig gebaut, wohI genährt und von gesunder Gesichtsfarbe. Eine Anzahl von Warzen bedecken die Hände und Finger, welch letztere in leichter Beugestellung gehalten werden. A.n den Volae beider Hände ist die Epidermis rauh, gespannt, nicht auffällig schuppend, leicht gelblich getönt und zeigt die normalen Rillen und Beugefurchen in verstärktem Grade; dabei füblt sie sich rigider an

1) Die Kranke ist durch Herrn Prof. Riehl in der Sitzung der Wiener dermatologischen Gesellschaft am 14. Jänner 1903 demonstriert worden. 
als normal und ist deutlich verdickt; Rhagaden finden sich nicht vor, sollen auch nie bestanden haben. Am Thenar, Hypothenar und Handgelenk geht die erkrankte Haut allmählich ohne scharfe Grenze, ohne roten Saum in die normale Haut über. An den Fingern setzt sich die Affektion auf der Beugeseite bis zur Mitte der Endphalangen fort, so zwar, daß die Gelenkbeugen annähernd normal und weniger gelblich getönt erscheinen. Interdigitalfalten und -Flächen sind normal, der Übergang zwischen erkrankter und gesunder Haut ein allmählicher, ohne entzündliche Erscheinungen. Die Streckseiten beider Hände, einschließlich der Finger, sind eyanotisch verfärbt und fühlen sich kalt an. Von den Nägeln macht der des rechten Daumens eine Ausnahme: er ist dünner als die übrigen, durch Längsfurchen und -Riffen stark deformiert, zeigt aber normale Rosafärbung und, wenn man den stark vorgeschobenen Nagelfalz zurückarängt, eine in normaler Weise nach vorn konvex begrenzte Lunula. An allen übrigen Nägeln ist die Luuula nicht als solche erkennbar, es ist vielmehr ihre stelle, sowie eine mehr oder weniger große anstoßende Partie der freien Nageloberfläche bläulichweiß verfärbt. Diese Verfärbung greift meistens an den seitlichen Rändern etwas weiter distalwärts vor, so daß als Begrenzung der weißen Fläche gegen die normal rosa gefärbte distale Partie eine nach vorn konkave Linie erscheint, die jedoch nicht derart scharf sich präsentiert wie die Lunula-Linie, indem ein mehr allmählicher Übergang zur Rosafärbung stattfindet. Die weißlich verfärbten Stellen nehmen ca. $2 / 5$ bis $2 / 3$ des ganzen Nagels ein. Außerdem finden sich aber in den distalen rosagefärbten Nagelpartien etwa 1-1-1/2 $\mathrm{mm}$ breite, genau querverlaufende, gerade, manchmal durch feine rosa Streifen segmentierte kreideweißschimmernde bandförmige Streifen, die sich gegen die normale rosafarbene Umgebung vielfach nicht scharf absetzen, sondern in feine Strichelchen und Pünktchen auflösen. Solcher Bänder finden sich $1-3$ in den verschiedenen Nägeln vor; wo mehrere von ihnen parallel verlaufen, sind sie durch ebenfalls ca. $1 \mathrm{~mm}$ breite normale, rosafarbene Streifen getrennt. Die Wölbung der Nägel ist, vom rechten Daumen abgesehen, normal, ihre Oberfläche glatt, glänzend, ohne Furchen und Riffen; der freie, beschnittene Rand blättert nicht auf; unter ihm erscheint der Übergang vom Nagelbett zur Epidermis der Fingerkuppe etwas verdickt, aber nicht schuppend oder rhagadisiert. Die Nägel der Füße sind infolge unzweckmäßigen Schuhwerks etwas deformiert, zeigen aber keinerlei weiBliche Verfärbungen. Auf den Plantae haben sich einige Druckschwielen gebildet, das Fußgewölbe bat jedoch weiche, glatte, normale Haut. Die Haare sind ohne Besonderheiten, seit einiger Zeit besteht trockne seborrhoische Schuppung. Pigmentations-Anomalien anderer Körperteile bestehen nicht. An beiden Oberarmen und Oberschenkeln ist Lichen pilaris deutlich ausgeprägt; unterhalb beider Patellae und über beiden Ellenbogen sind ziemlich stark ichthyotische Plaques. Die Zähne sind wohlgebildet.

Zur mikroskopischen Untersuchung konnten erklärlicherweise leider auch nur dem freien Rand entnommene Nagel- 
schnitzen verwendet werden, doch traf es sich glücklicherweise so, daß einige Stücke der oben beschriebenen breiten weißen Streifen gewonnen wurden. Diese kamen nacheinander in Alkohol steigender Konzentration, Xylol und Paraffin von härterer Konsistenz, und so gelang es Schnitte zu erzielen, in denen keine Quellung der Hornsubstanzen durch Alkali oder Säuren stattgefunden hatte. Es fanden sich im Querschnitte bei durchscheinendem Licht zwei dunklere Zonen parallel zur Oberfläche verlaufend, die eine schmäler und dicht unter der Oberfï̈che gelegen, die andere ungefähr in der Mitte des Schnittes und dessen ganzes mittleres Drittel einnehmend. Beide setzen sich aus feineren und gröberen Punkten und Strichen zusammen, lassen aber auch in ihrem Zentrum größere, anscheinend homogene Partien erkennen, die sich bei stärkerer Vergrößerung auch in dicht beieinander stehende Punkte, Striche und Flecke auflösen. Im Längsschnitt ist gegen den freien Nagelrand zu eine geringe Senkung der dunkleren Partien nach der Unterfläche des Nagels zu konstatieren. Bei auffallendem Lichte betrachtet, werden die nämlichen Stellen weißlich leuchtend und heben sich von der dunklen Umgebung gut ab, einem Nebelfleck am Nachthimmel vergleichbar, der sich bei starker Vergrößerung in einzelne Sternchen auflöst.

Bei Färbung mit Hämalaun-Eosin nimmt die gesunde Nagelsubstanz das Eosin ziemlich diffus auf, leicht dunklere punktförmige Fleckchen lassen nur stellenweise die zu Grunde gegangenen Kerne ahnen. Dagegen hat sich der Streifen im oberen Teile des Nagels mit Hämalaun violett-schwarz tingiert und zeigt bei starker Vergrößerung plattgedrückte Kernformen; andererseits ist an dem Streifen im mittleren Drittel des Nagels nur geringe Hämalaunfärbung zu sehen, er hat vielmehr größtenteils die gelblich-braune Tönung des ungefärbten Präparates konserviert. Dementsprechend findet man auch, wenn jetzt dies Präparat im auffallenden Lichte betrachtet wird, den mittleren Streifen stark leuchtend, während die Region des oberen Streifens dunkel bleibt.

Bei einem nach van Gieson gefärbten Schnitte ist der obere Streifen braunrot bis dunkelbraun gefärbt, läßt ebenfalls bei starker Vergrößerung dunkle plattgedrückte Kerne noch teilweise unterscheiden und leuchtet im auffallenden Lichte nicht; der in der Nagelmitte gelegene Streifen ist schwächer rot gefärt, läßt keine dunkleren Kerne erkennen, wohl aber stärker gelbliche Zonen, wo dann die bräunlichen Pünktchen des ungefärbten Präparates wieder deutlicher sichtbar werden. Die übrige gelb gefärbte normale Nagelsubstanz zeigt eine feine schwach rötlich gefäbte Maserung. Demgemäß erscheint auch in diesem Präparat bei auffallendem Lichte der mittlere 
Teil des Schnittes leuchtend, der obere Streifen dagegen mit Ausnahme weniger allerfeinster Pünktchen dunkel.

Somit bestätigt unser mikroskopischer Befund die Angabe früherer Autoren den Luftgehalt der erkrankten Nägel betreffend; es erhellt aber auch gleichzeitig aus ihm, daß in der Tat an den Stellen, wo das ungefärbte Präparat Lufträume erkennen läßt, eine Parakeratose besteht, resp. bestanden hat. Sie besteht in unseren Präparaten im oberen Streifen, denn dort sehen wir mehr oder minder deutliche Kerntinktion mit Hämalaun, wodurch im Gieson-Präparat der bräunlichrote Ton entsteht. Sie hat bestanden und ist größtenteils zum Abschluß gelangt in der mittleren Partie, wo infolge Absterbens der Kerne Hämalaun fast nicht mehr aufgenommen wird, und wo bei auffallendem Lichte im Hämalaun-Eosin-Präparat die Luft wieder weißlich schimmert, wie im ungefärbten. Daß diese Partie im Gieson-Präparat durch Fuchsin gefärbt erscheint, dürfte wohl so zu erklären sein, daß die Säurefuchsinpikrinlösung in die Lufträume eingedrungen, deren Wandungen durch Säurewirkung zum Quellen brachte und so in diesen festgehalten wurde.

Betrachten wir also die Parakeratose, die ja auch Jos e ph und Heidingsfeld bereits gesehen haben, und mit ihr die Ernährungsstörung als feststehende Tatsache, so ist doch die Ätiologie der Erkrankung noch nicht geklärt. Die Erfrierungen, welche unsere Patientin in wiederholten Schüben durchgemacht hat, können vielleicht als die Veranlassung dieser Ernährungsstörung angesehen werden; gleichwohl sind wir nicht gezwungen, sie für ausreichend zur Erklärung des Prozesses in unserem Falle zu halten. Denn wie viele erfrorene Hände und Füße gibt es, und wie wenig Fälle von Leukonychie sind bekannt geworden! Es muß noch ein anderes Etwas mitgewirkt haben. Diese Empfindung hat auch schon Heller gehabt, als er für die durch Trauma entstandenen Flores unguium eine „gewisse Disposition" annehmen zu müssen glaubte. In unserem Falle ist die Disposition vorhanden; denn es besteht bei der Patientin eine Ichthyosis simplex mäßigen Grades, die den Angaben der Patientin gemäß nach der ersten Erfrierung der Hände sich auch an diesen in den Beugeflächen lokalisiert haben soll. Wenn also die Erfrierung allein keinen ausreichenden Grund für die Entstehung der Leukonychie gibt, so ist es nicht von der Hand $\mathrm{zu}$ weisen, daß die Konkurrenz derselben mit Ichthyosis 
zu dieser Störung der Nagelbildung geführt hat. Zwar waren keine direkt wahrnehmbaren Erscheinungen der Ichthyosis an den Streckseiten der Phalangen sichtbar, doch müssen wir nach anderweitigen klinischen Erfahrungen die gesamte Epidermis ichthyotischer Individuen als abweichend von der normalen ansehen. Auffällig ist es, daß der durch ein früheres Panaritium deformierte rechte Daumennagel an der Erkrankung der übrigen Nägel nicht teilgenommen hat. Was den Punkt anbetrifft, daß die Patientin das Weißwerden der Nägel erst in den letzten zwei Monaten bemerkte, so bezieht sich diese Angabe natürlich nur auf die konfluierende Verfärbung am Nagelgrunde. Denn die mehrfachen Querstreifen, die wir mit Stout als Vorstadien der totalen Leukonychie ansehen, ohne damit sagen zu wollen, daß der Prozeß nicht in diesem Stadium dauernd verharren könne, haben bereits den freien Nagelrand erreicht und beweisen damit, daß schon seit langer Zeit schubweise intermittierend - ernährungsstörende Einflüsse gewirkt haben, die nun endlich die Matrix in bestimmten Partien dauernd verändert haben. Es haben also wahrscheinlich die Erfrierungen der früheren Jahre nur nicht weiter beachtete weiße Fleckchen und Streifchen geschaffen, und erst die neverliche Erfrierung zu Beginn des letzten Winters setzte an Stelle des intermittierenden den dauernden Zustand von Unterernährung. Gelegentlich seiner Demonstration teilte Prof. Riehl mit, daß er noch drei andere Fälle von Leukonychie beobachtet habe, einen totalen und zwei, bei denen größere Abschnitte der Nägel von der Verfärbung betroffen waren, und betonte, daß alle drei Patienten an Erfrierungen der Hände gelitten haben, so daß an einen möglichen Zusammenhang ron Leukonychie und Erfrierung zu denken sei.

Prüfen wir jetzt, soweit die einzelnen Berichte dies ermöglichen, die in der Literatur bekannt gewordenen Fälle von Leukonychie auf das Vorhandensein von Disposition und Ernährungsstörung, so finden wir bei Unna die Mitteilung, daß sein Tischler etwas cyanotisch gefärbte Hände hatte "wie bei leichtem Frost". Die "breite, festonartig ausgehöblte subunguale Hornschicht", die der Autor selbst als stark hypertrophische Hornschicht des Nagelbettepithels auffaßt, läßt unwillkürlich an eine ichthyotische Disposition denken. Besonders merkwürdig ist mit Beziehung zu unserem eigenen Falle der Umstand, daß nach einor Verletzung die beschädigten beiden Mittelfinger zuerst rote Nägel erhielten, die später 
erst wieder weiß wurden. Muß man da nicht annehmen, daß zur Reparatur des gesetzten Schadens eine zeitweilige Überernährung eingetreten ist, bei der die vorhandene Disposition allein keine Wirkung ausüben konnte? Morisons junge Dame zeigte ihre Leukonyehia striata nur im Winter, im Sommer blieb der Nachschub aus. Bei ibr können wir als allgemeine Disposition ihre starke Anämie ansehen, die mit jeden Menses eine kurze lokale Steigerung erfahren haben dürfte, womit das Auftreten der Streifen in 3-4wöchentlichen Intervallen zwanglos erklärt wäre. Stouts Mulatte war skrofulös veranlagt und litt an chronischer Gastritis. Bei Giov vannini s Kutscher folgte die Erkrankung auf ein typhöses Fieber; über eine besondere Disposition ist aus den Angaben nichts zu eruieren. JosephsSchlächter hatte beim Beginn der Affektion Schwellung und Schmerz. haftigkeit der distalen Fingerteile bemerkt. Hier ist $\mathbf{z u}$ berücksichtigen, daß die Zeit der Beobachtung (Feber-März), sowie seine Beschäftigung (in den Kühlräumen des Schlachthauses, vielleicht auch mit Eisstücken?) die Vermutung einer Erfrierung nahe legt; Angaben, aus denen auf eine bestimnate Disposition zu schließen wäre, fehlen. Longstreth beobachtete an sich selbst jedesmal nach einem Anfall von Rückfallfieber das Auftreten weißer Bänder auf den Nägeln; ihm fehlte jedenfalls die Disposition, sonst würde er bleibende Streifen wie Morisons Patientin bekommen haben. Bei Bielschowskys Falle von multipler AlkoholNeuritis, mit folgendem Auftreten weißer Streifen quer über die Nägel, muß unseres Erachtens auch der voransgegangene Gastroenterokatarrh als ätiologisches Moment mit in Frage gezogen werden. Nach Langdon Down wurde bei einer Prostration infolge von Überarbeitung unregelmäßige Herzaktion beobachtet, und später folgte eine Leukonychia punctata. Der Patient, den Parkes Weber and Krieg in der Londoner dermatologischen Gesellschaft demonstrierten, litt an einem Herzklappenfehler; außerdem aber zeigte er beginnende Koilonychie, mit subungualer Hyperkeratose, und der Nagelfalz war von der Nagelplatte etwas abgehoben, obwohl der Patient ihn nicht zurückzuschieben pfiegte. Der zweite von Forchheimer bearbeitete Fall Josephs betraf ein 17jähriges stark anämisches, vielleicht auch rhachitisches Mädchen, bei dem subunguale Keratose und Koilonychie bestand. Es fehlt ein Anhaltspunkt, welches der ursächliche Reiz zur Entstehung der Leukonychie gewesen; soviel erscheint klar, daß derselbe bei zwei disponierenden Zaständen nur ein minimaler zu sein braucht, um die Hyperkeratose zur Parakeratose umzugestalten. Lawrence sah Leukonychia striata bei Vater und Sohn und schloß daraus auf Vererbung. Heller (21jähriger Bäcker), Bergmann (9jähriges Mädchen) und Colombini (32jähriger Mann) können keinerlei ätiologische Notizen geben, Heidingsfelds 7 Fälle sind traumatischen Ursprungs.

Wir sehen also, daß es gelingt, in einer Anzahl der Schilderungen eine Disposition nachzuweisen; und zwar handelt es sich vorzugsweise um Hyperkeratose, die durch die Er- 
nährungsstörung eine Umsetzung in Parakeratose erfährt, oder um Anämie, die - wie man z. B. an den sogenannten anämischen Ekzemen sieht - eine Parakeratose begünstigt. Dje Art der ernährungsstörenden Momente kann sebr mannigfaltig sein: Congelatio, Neuritis, Gastritis, Typhus, Recurrens, Vitium cordis, Trauma der Matrix; sie ist untergeordneter Natur. Das Wesen der Leukonychie sowohl als der Leukopathia unguium haben wir in der Parakeratose zu sehen, und es ist daher nicht berechtigt, beide trennen zu wollen, wie dies Joseph tut, der jede als "Erkrankung sui generis" ansieht. ${ }^{1}$ ) Der Fall S touts z. B. ist ein Übergang einer Leukonychia punctata zur maculata und striata; ebenso bildet Hellers Bäcker- und BergmannsMädchen ein solches Beispiel und endlich zeigt auch unser eigener Fall verschiedene Formen. Besser wäre es daher nicht nach der Form eine Leukonychia punctata, maculata, striata, partialis, totalis zu unterscheiden, sondern nach dem Erscheinungsmodus eine Leukonychia acuta oder simplex und eine Leukonychia chronica oder perstans, deren jeder man noch das formbezeichnende Epitheton beigeben könnte.

1) Dermatolog. Zeitschrift. Bd. V. pag. 654. 


\section{Literatur.}

Bergmann. Die Krankheiten der Haut, Haare und Nägel am menschlichen Körper. Leipzig 1842. Zitiert bei Heller.

Bielschowsky. Beitrag zur Lehre von den trophischen Veränderungen der Nägel bei multipler Neuritis. Neurologisches Zentralbl. Band IX, pag. 741 .

Colombini. Un caso di leuconichia. Riforma medica. 2./VII. 1894. Nr. 151. pap. 2.

Forchheimer. Ein Fall von Leukonychie, verbunden mit Koilonychie. Dermatol. Zentralbl. Bd. II. pag. 33. cf. Joseph 2.

Giovannini. Canities unguium. Internationaler Atlas seltener Hautkrankheiten. Heft 6, Tafel XIX, 1.

Heid ingsfeld. Leukopathia unguium. Journal of cut. and gen.urin. diseases 1900. pag. 490.

Heller. Die Krankheiten der Nägel. Berlin 1900.

Joseph. 1. Leukonychia. Neissers stereoskopischer Atlas, Tafel 199. - 2. Demonstrationen in der Berliner dermatologischen Gesellschaft am 14. Juni 1898. Dermatologische Zeitschrift. Band V. pag. 651. cf. Forchheimer.

Lawrence. Leukopathia. The Australian medical Journal. 15./X. 1893. Zitiert bei Heller \& Stout.

Langd on Down. Transact. of the Patholog. Society 1870. Band XXI. pag. 409. Zitiert bei Stout.

Longstreth. On changes in the nails in fever and especially in relapsing fever. Zitiert bei Stout.

Morison. Leukopathia unguium. Archiv für Dermatologie und Syphilis. Band XX. pag. 3 .

Parkes Weber und Krieg. Demonstration in der Londoner dermatologischen Gesellschaft. British Journal of Dermatology. Band XI. pag. 120.

S t o ut. Leukopathia unguium. Philadelph. medic. News. 24./П. 1894. Band LXIV. pag. 212.

Unna. Leukonychia und Leukotrichie. Internation. Atlas seltener Hautkrankheiten. Heft 6. Tafel XIX, 2. 


\section{Nachtrag.}

Im Anschluß an vorstehende Arbeit sei noch erwähnt, daß neuerdings ein 9jähriger Knabe zur Beobachtung kam, der seit 4 Jahren an erfrorenen Händen leidet und dabei eine an den Knien und Ellenbogen deutlich ausgeprägte Iehthyosis simplex zeigt. Bei ihm waren sämtliche Nägel der Finger von mehr oder minder großen weißen Flecken durchsetzt, deren größte $1 / 2 \mathrm{~cm}$ breit und $3 / 4 \mathrm{~cm}$ lang waren. Ferner saben wir in der Ambulanz ein 22jähriges Mädchen mit eine an Erythema multiforme erinnernden Affektion beider Hände und Ichthyosis simplex über den Knien und Lichen pilaris an den Streckseiten der Oberarme. Dieses Erythem der Hände war innerhalb Jahresfrist $7 \mathrm{mal}$ rezidiviert, auch im Sommer, and hatte je etwa 14 Tage angehalten. Die Nägel aller Finger hatten eine mit der Lunula ohne Grenze verbundene milchweiße Verfärbung, die sich mehr oder weniger weit distal, vereinzelt bis fast zum freien Nagelrand erstreckte. Gleichzeitig bestand subunguale Keratose und beginnende Koilonychie. Endlich stellte sich ein 16jähriger junger Mann vor, der vor 3 Monaten sich zum ersten Male die Hände erfroren hatte; er zeigte an mehreren Nägeln Leukonychia punctata. Bei genauer Inspektion ergab sich Ichthyosis simplex über beiden Knien und Ellenbogen, ferner leichte subunguale Keratose und Rhagadenbildung der Nagelfälze.

\section{Die Erklärung der Abbildungen auf Taf. IV ist dem Texte zu entnehmen.}


Archiv f. Dermatologie u. Syphilis Band LXVII.

TAF IV.

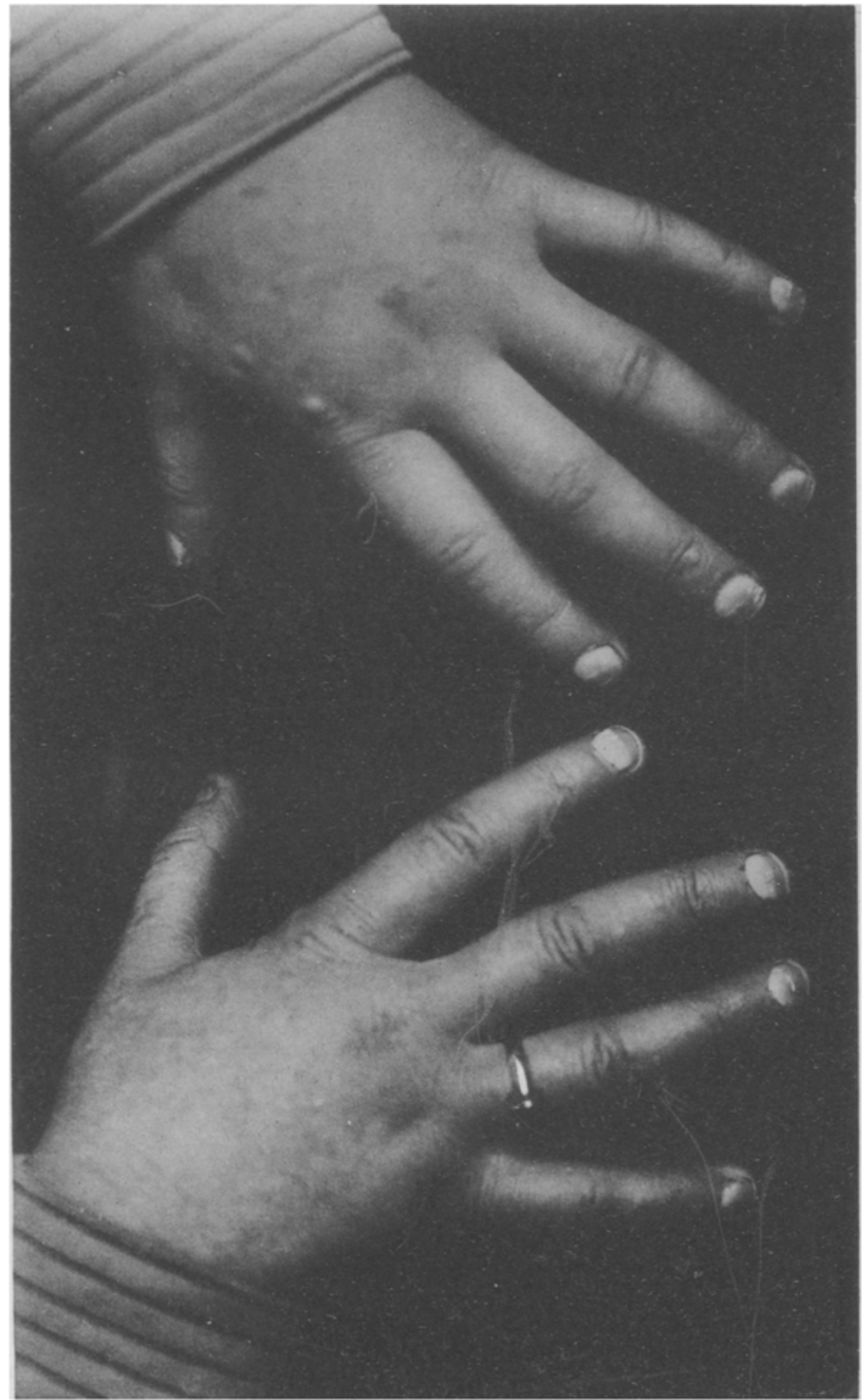

Biauns: Zur Kenntris der Leukonychie.

K.4. K. Hethithagr. A.Mazse.frig. 